Case Report

\title{
An Atypical Presentation of Myopericytoma in Palmar Arch and Review of the Literature
}

\author{
Adnan Kara, Mert Keskinbora, Mahmut Enes Kayaalp, Ali Şeker, \\ Mehmet Erdil, and Murat Bülbül \\ Department of Orthopaedics and Traumatology, Faculty of Medicine, Istanbul Medipol University, Istanbul, Turkey \\ Correspondence should be addressed to Adnan Kara; dradnankara@gmail.com
}

Received 21 July 2014; Accepted 21 September 2014; Published 2 October 2014

Academic Editor: Hiroshi Hatano

Copyright (C) 2014 Adnan Kara et al. This is an open access article distributed under the Creative Commons Attribution License, which permits unrestricted use, distribution, and reproduction in any medium, provided the original work is properly cited.

Introduction. Myopericytoma is a very rare perivascular tumor that can be presented with painful mass in lower extremities. We aimed to present an atypical presentation and location of myopericytoma. Presentation of Case. An 18-year-old otherwise healthy individual was admitted to outpatient clinic with complaints of numbness and pain in his right hand. He has had no trauma. On volar aspect of his right hand, a well-circumscribed, painful mass was palpated. MRI results were related to hemangioma. Surgical excision was planned and performed. Pathological investigation revealed the mass is myopericytoma. Discussion. This case demonstrates a rare location and presentation of myopericytoma. Reviewing the literature, discussion was made to expand the horizon for diagnosis and treatment of patients with similar symptoms. Conclusion. Myopericytoma can rarely present with numbness and pain in affected region. Surgical excision is helpful for definitive diagnosis and symptom relief.

\section{Introduction}

Myopericytomas are perivascular benign neoplasms. These uncommon lesions are believed to arise from perivascular myoid cells [1]. They are seen most frequently in lower extremities in the dermal or subcutaneous location [13]. A myopericytoma patient typically presents with wellcircumscribed, slow growing, painless firm mass [2].

In this paper, we present 18 -year-old male patient with a painful mass in thenar region of his right hand. He had also numbness in ulnar side of his thumb. We aimed to discuss this atypical presentation of this rare entity in the light of the literature.

\section{Case Report}

An 18-year-old male patient was admitted to our outpatient clinic with complaints of ongoing pain in his thenar region and numbness of 6-month duration in ulnar side of his right thumb. In the physical examination, a $3 \times$ $2 \mathrm{~cm}$ well-circumscribed, painful mass in his thenar region was seen. His past medical history and family history were unremarkable. Preoperative complete blood count, erythrocyte sedimentation rate, and C-reactive protein values were within normal ranges. Preoperative $\mathrm{X}$-ray examinations were normal. Preoperative MRI demonstrated a lobulated, wellcircumscribed, contrast enhanced mass, which was $24 \times$ $20 \times 16 \mathrm{~mm}$ in size and had low signal intensity in T1A (Figure 1(a)) and high signal intensity in T2A (Figure 1(b)). These findings were found relevant to hemangioma.

Under tourniquet control, we perform a curved incision over the mass in the thenar region of the right hand. With meticulous dissection of soft tissues, the margins of the lesion were exposed between adductor pollicis and flexor pollicis brevis muscles. Superficial palmar arch was also exposed during the dissection. Under the lesion, we observed the compression of the lesion to the ulnar side of palmar digital nerve with some adhesions to the lesion. We excised the lesion from the nerve under microscopic dissection (Figure 2).

The excised lesion was prepared for histopathological evaluation.

Patient reported relief of pain and numbness symptoms at the 4 th week of the surgery. At the last follow-up of the 14 th 


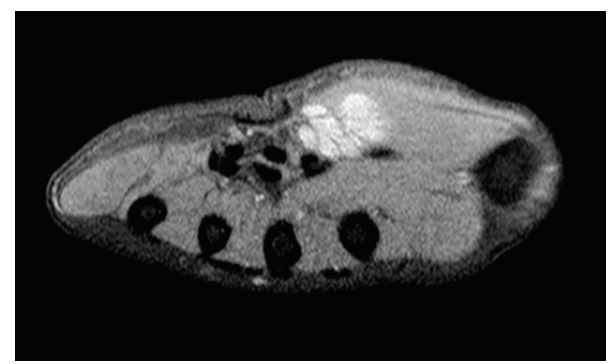

(a)

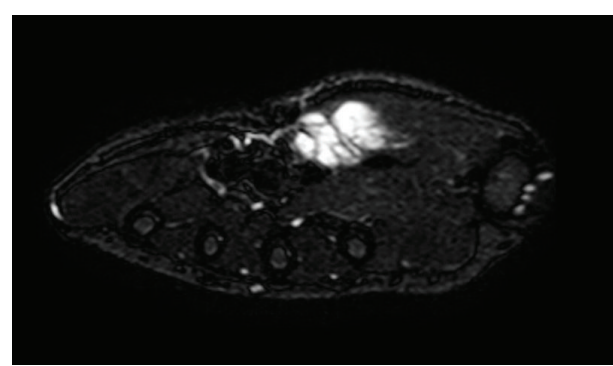

(b)

FIgURE 1: (a) Low signal intensity in T1A. (b) High signal intensity in T2A.

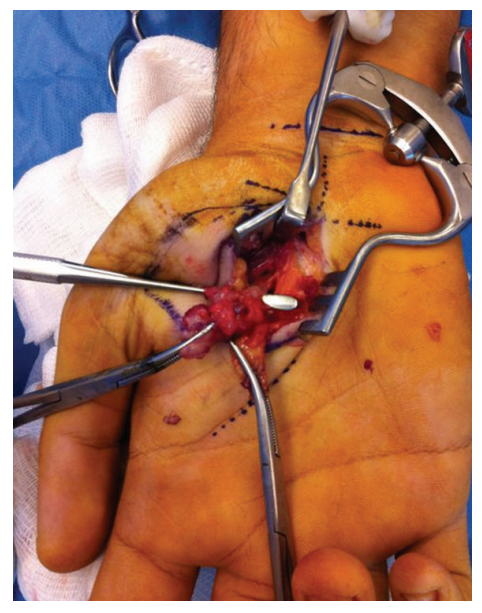

FIGURE 2: Lesion was excised with microscopic dissection.

month after surgery, the patient was well with no recurrence or symptoms.

\section{Discussion}

Myopericytomas are uncommon benign perivascular neoplasms that show a hemangiopericytoma-like vascular pattern $[1,2,4,5]$. Patients with myopericytoma are frequently presented with well-circumscribed, slow growing, painless firm mass $[2,3]$. In contrast, our patient complained of a painful lesion in his right hand with numbness radiating to the ulnar side of his thumb. We observed the compression of the lesion to the ulnar side of palmar digital nerve during dissection. At the 4 th week of surgery, numbness and pain complaint were relieved.

These lesions frequently arise in lower extremities, whereas they can be found in upper extremities, head and neck region, and the trunk $[1,4]$. To the best of our knowledge, there are only two cases of hand localization of myopericytoma $[6,7]$. In our patient, the lesion was in the thenar region of his right hand.

According to the literature, preoperative MR and ultrasound investigations are found to be insufficient or misleading $[6,8]$. Excisional biopsy and histological examination are necessary for definitive diagnosis. Also in our case, preoperative MRI reported the case as hemangioma.
The histopathological examination revealed CD34 expression in endothelial cells and thin-walled vessels with a concentric, perivascular gathering of spindled myoid tumor cells as similarly described in the literature (Figure 3(a)). These cells stain positive for SMA (smooth muscle actin) and often also for caldesmon, are immune negative for desmin, and are accepted as modified smooth muscle cells $[1,6]$ (Figure 3(b)). The same results were obtained in our case. Ultrastructurally, myopericytes have a transitional phenotype and are intermediate cells between pericytes and vascular smooth muscle cells [9].

Recurrent and/or malignant cases of myopericytomas are rarely reported, even though they are excised marginally or incompletely. Additionally, some local recurrences are reported in a few numbers $[4,5,10-12]$. In our case, no proof of recurrence was noticed at the 14th month of follow-up.

Surgical excision is the preferred treatment method. Several studies show good-to-excellent results with low rate of local recurrence $[4,7]$. In our case, we performed surgical excision with meticulous dissection of nerve and vessels.

\section{Conclusion}

Myopericytomas are rare tumors that can show atypical presentations. Surgical excision is the gold standard for diagnosis and treatment. 


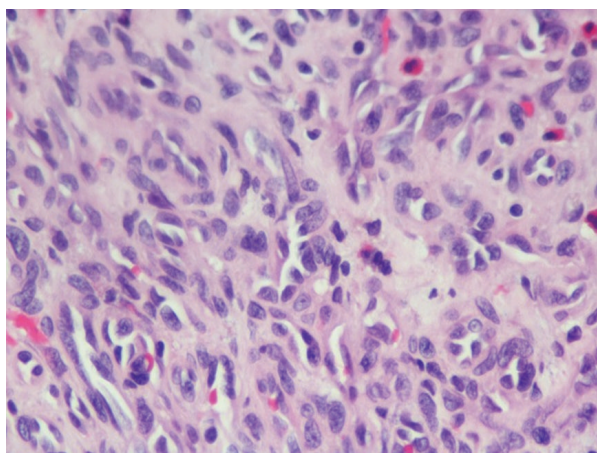

(a)

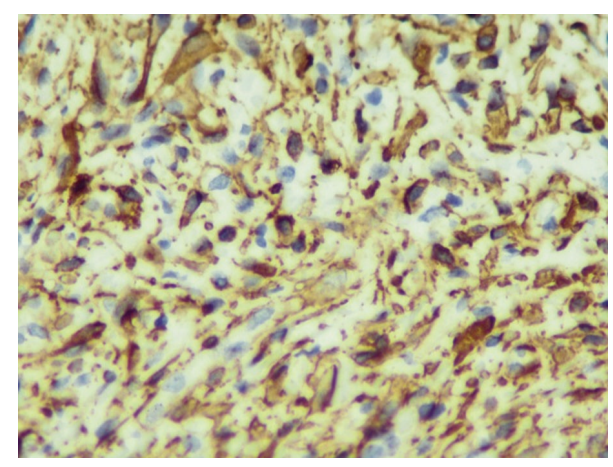

(b)

Figure 3: (a) Thin-walled vessels with a concentric, perivascular gathering of spindled myoid tumor cells (HE-Stain, $\times 40)$. (b) Pericytes stained positive for caldesmon.

\section{Conflict of Interests}

The authors declare that there is no conflict of interests regarding the publication of this paper.

\section{References}

[1] F. Ide, K. Obara, H. Yamada, K. Mishima, and I. Saito, "Intravascular myopericytoma of the oral mucosa: a rare histologic variant in an uncommon location," Virchows Archiv, vol. 450, no. 4, pp. 475-477, 2007.

[2] M. E. McMenamin and E. Calonje, "Intravascular myopericytoma," Journal of Cutaneous Pathology, vol. 29, no. 9, pp. 557561, 2002.

[3] S. R. Granter, K. Badizadegan, and C. D. M. Fletcher, "Myofibromatosis in adults, glomangiopericytoma, and myopericytoma: a spectrum of tumors showing perivascular myoid differentiation," The American Journal of Surgical Pathology, vol. 22, no. 5, pp. 513-525, 1998.

[4] T. Mentzel, A. P. Dei Tos, Z. Sapi, and H. Kutzner, "Myopericytoma of skin and soft tissues: clinicopathologic and immunohistochemical study of 54 cases," American Journal of Surgical Pathology, vol. 30, no. 1, pp. 104-113, 2006.

[5] E. Calonje, "Vascular tumors: tumors and tumor-like conditions of blood vessels and lymphatics," in Lever's Histopathology of the Skin, D. E. Elder, R. Elenitsas, B. L. Johnson Jr., G. F. Murphy, and X. Xu, Eds., pp. 1049-1051, Lippincott-Raven, Philadelphia, $\mathrm{Pa}$, USA, 10th edition, 2008.

[6] A. C. Woollard, C. Southgate, and J. W. Blair, "Intravascular myopericytoma of the superficial palmar arch," Journal of Hand Surgery (European Volume), vol. 32, no. 4, pp. 475-476, 2007.

[7] P. Mahapatra, J. Dunne, and R. J. Colville, "Digital artery intravascular myopericytoma-a rare cause of a painful finger," Journal of Hand Surgery (European Volume), 2014.

[8] Y. Uchida, M. Kuriyama, Y. Yoshida, A. Yano, and K. Orihashi, "Diagnosis and surgical management of the arterial myopericytoma," Journal of Plastic, Reconstructive and Aesthetic Surgery, vol. 65, no. 7, pp. e200-e201, 2012.

[9] L. Díaz-Flores, R. Gutiérrez, M. P. García, L. Díaz-Flores Jr., F. Valladares, and J. F. Madrid, "Ultrastructure of myopericytoma: a continuum of transitional phenotypes of myopericytes," Ultrastructural Pathology, vol. 36, no. 3, pp. 189-194, 2012.
[10] S. Sapelli, M. Ribas, W. D. Martins, L. de Noronha, and A. P. Gomes, "Myopericytoma of the lip: report of case," Head and Neck, vol. 31, no. 4, pp. 561-564, 2009.

[11] M. E. McMenamin and C. D. M. Fletcher, "Malignant myopericytoma: expanding the spectrum of tumours with myopericytic differentiation," Histopathology, vol. 41, no. 5, pp. 450-460, 2002.

[12] A. C. Laga, A. L. Tajirian, M. N. Islam et al., "Myopericytoma: report of two cases associated with trauma," Journal of Cutaneous Pathology, vol. 35, no. 9, pp. 866-870, 2008. 


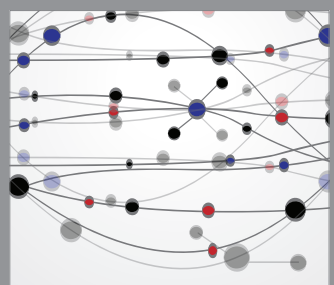

The Scientific World Journal
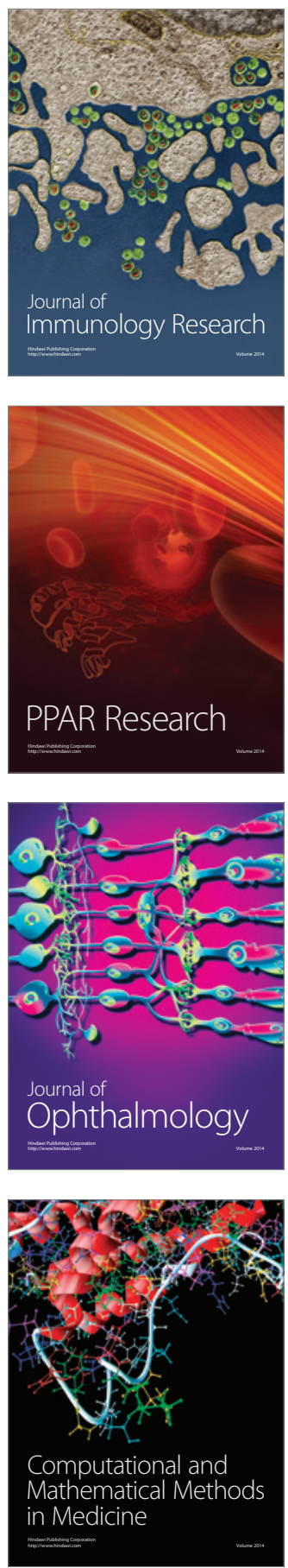

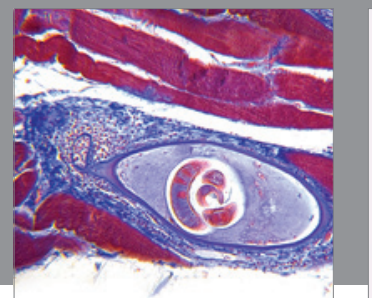

Gastroenterology

Research and Practice
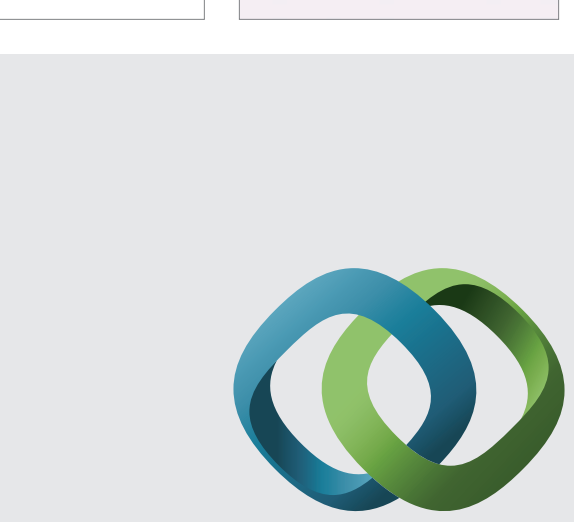

\section{Hindawi}

Submit your manuscripts at

http://www.hindawi.com
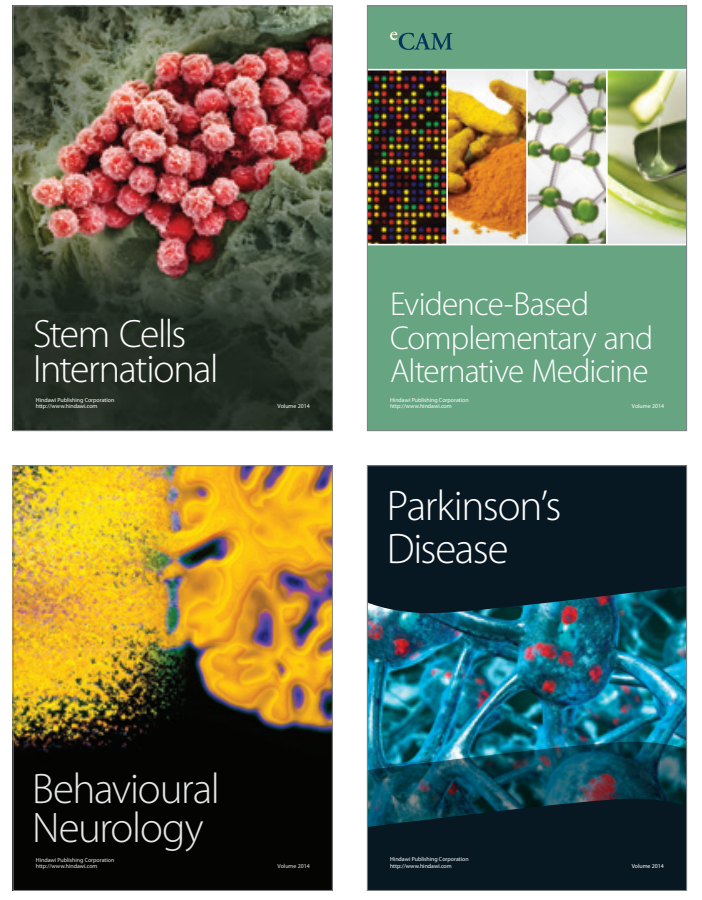
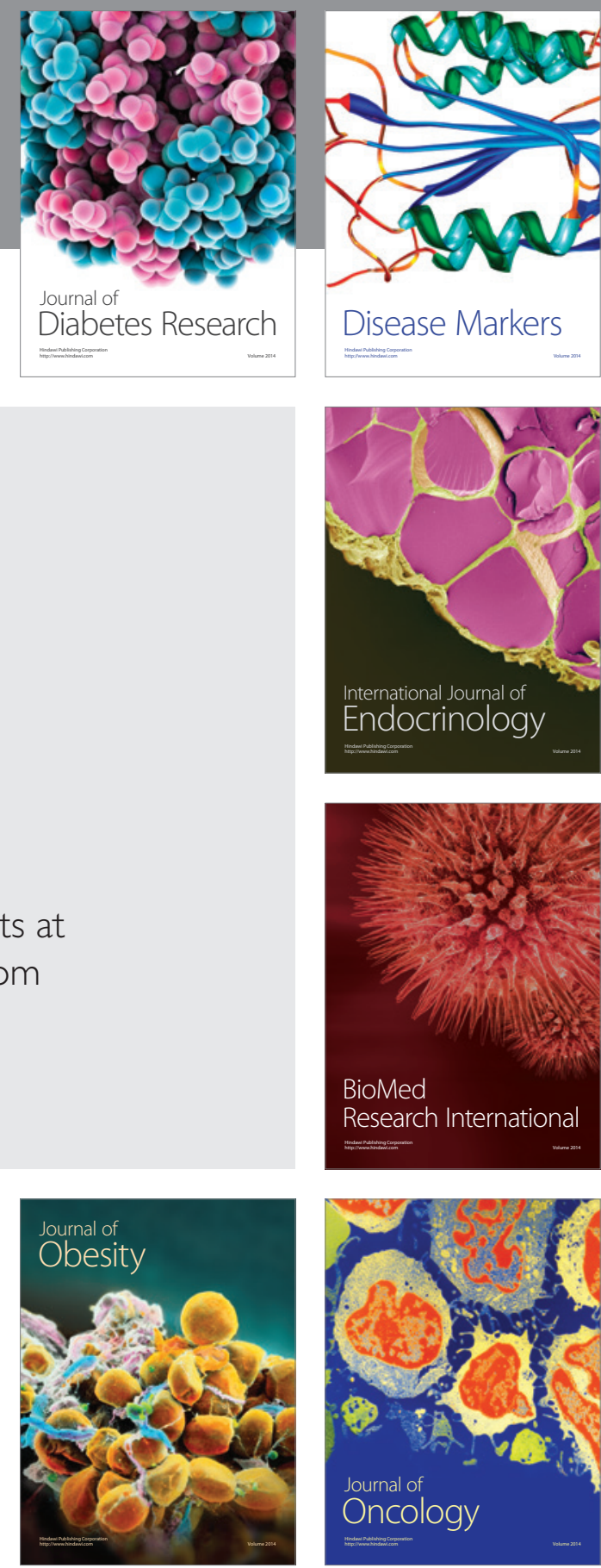

Disease Markers
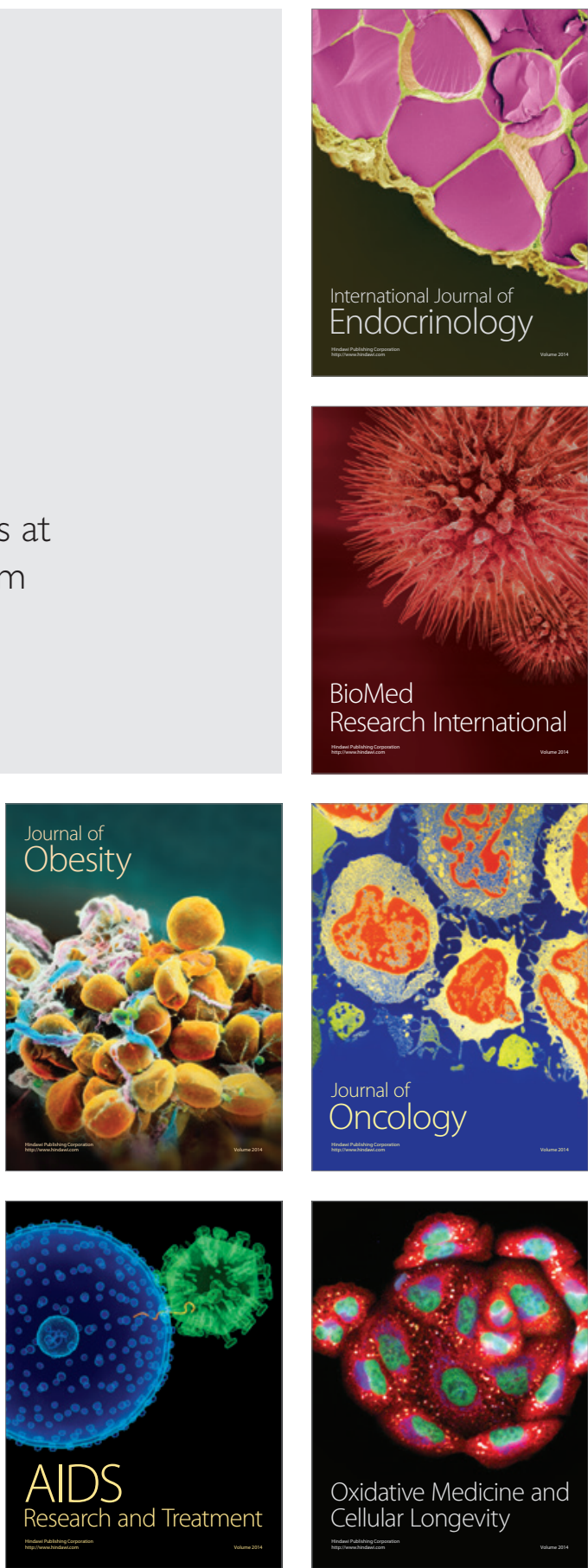